\title{
Confluent and Reticulated Papillomatosis of Carteaud and Gougerot in a Young Nepali Male
}

\author{
Amatya $B^{1}$, Sharma $R^{2}$, Syed MMA², Rajbhandari $A^{3}$ \\ ${ }^{1}$ Lecturer, Department of Dermatology and Venereology, Nepal Medical College and Teaching Hospital, Kathmandu, \\ Nepal; ${ }^{2}$ Resident, Department of Dermatology and Venereology, Institute of Medicine, Tribhuvan University Teaching \\ Hospital, Maharajgunj, Kathmandu Nepal; ${ }^{3}$ Resident, Department of Pathology, Institute of Medicine, Tribhuvan \\ University Teaching Hospital, Maharajgunj, Kathmandu Nepal
}

\begin{abstract}
Confluent and reticulated papillomatosis of Carteaud and Gougerot is a keratinization disorder with an infective aetiology. Patients present with hyperpigmented papules on the upper trunk and axillae that coalesce centrally and demonstrate reticulation peripherally. Diagnosis is based on clinical findings, characteristic histopathologic changes and response to therapy. We report a case of a young Nepali male who presented with gradual onset of asymptomatic raised dark brown lesions on his neck, trunk and axillae over the course of eight years. The condition was previously misdiagnosed as pityriasis versicolor and he had received oral and topical antifungals. The diagnosis was revised to confluent and reticulated papillomatosis based on clinical and histopathological examination. He was subsequently started on oral minocycline $50 \mathrm{mg}$ twice daily and nightly application of topical tretinoin $0.05 \%$ gel. There was complete resolution of all his lesions except for residual hyperpigmentation at the end of two months of therapy. There has been no relapse six months from the end of therapy. This is to our knowledge, the first case of confluent and reticulated papillomatosis reported from Nepal. Oral minocycline and topical tretinoin should be considered first line in the treatment of confluent and reticulated papillomatosis.
\end{abstract}

Key words: Hyperpigmentation; Minocycline; Nepal; Tretinoin

\section{Introduction}

$\mathrm{C}$ onfluent and reticulated papillomatosis (CRP) was first described by Gougerot and Carteaud in $1927 .{ }^{1}$ It is a rare condition that typically develops in young adults and presents with dark brown papules on the upper trunk and axillae that coalesce centrally and become reticulated peripherally. ${ }^{1}$ It is assumed to be a disorder of keratinization with an infective aetiology based on histopathological features and its response to antibiotics and retinoids. ${ }^{1}$ The treatment options include tetracycline and macrolide antibiotics, oral and topical retinoids, oral and topical antifungals and topical calcineurin inhibitors. ${ }^{1}$

Herein, we report a case of CRP in a young adult which

Financial disclosure: None.

Conflict of interest to disclosure: None declared.

Address of Correspondence

Dr. Bibush Amatya

Lecturer

Department of Dermatology and Venereology

Nepal Medical College and Teaching Hospital, Kathmandu, Nepal

E-mail: doc_bibush@hotmail.com, bibush.amatya@nmcth.edu responded to oral minocycline in combination with topical retinoid.

\section{Case Report}

A 23-year-old Nepali male presented to our hospital with a 7-year history of asymptomatic, brownish skin lesions in his neck, trunk and axillae. The lesions started on the trunk and slowly spread to the axillae and neck over several years. He had been treated with oral and topical antifungals in the past which did not lead to any improvement.

Submitted: $13^{\text {th }}$ September 2019

Accepted: $15^{\text {th }}$ November 2019

Published: $7^{\text {th }}$ October 2020

How to cite this article

Amatya B, Sharma R, Syed MMA, Rajbhandari A. Confluent and reticulated papillomatosis of Carteaud and Gougerot in a young Nepali male. Nepal Journal of Dermatology Venereology and Leprology. 2020;18(1):64-6. https://doi.org/10.3126/njdvl. v18i1.25594.

\section{(c) (i)}

Licensed under CC BY 4.0 International License which permits use, distribution and reproduction in any medium, provided the original work is properly cited. 


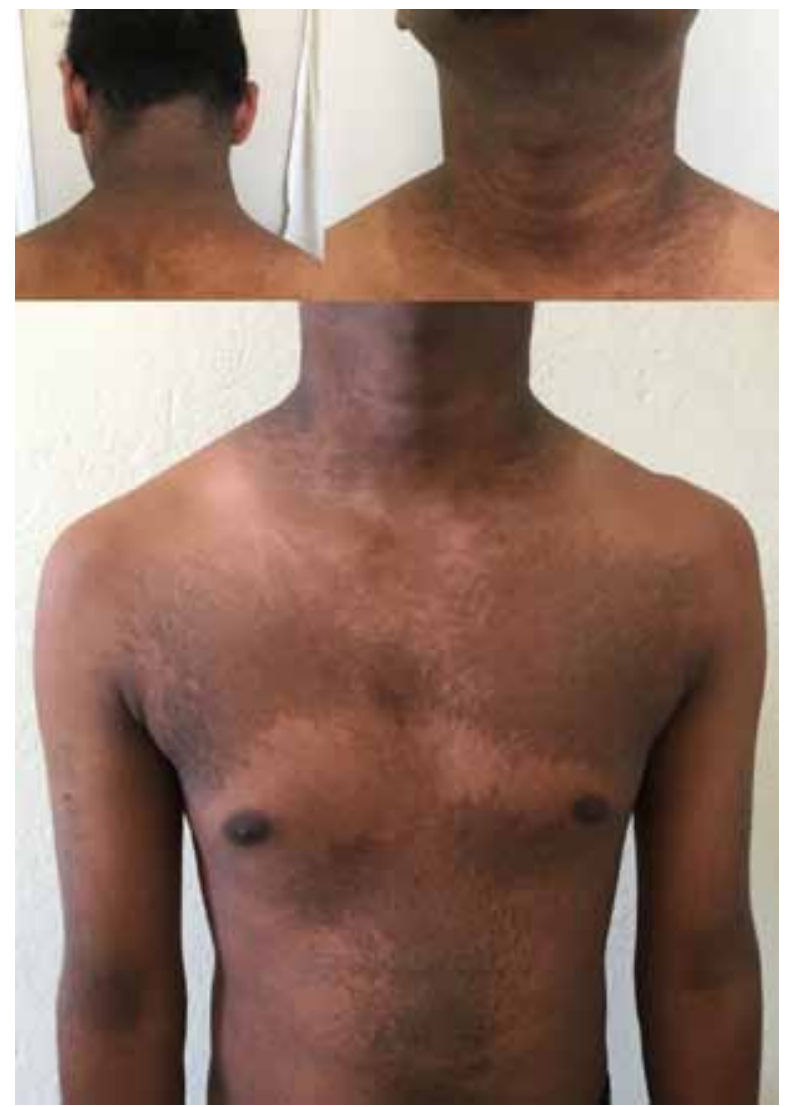

Figure 1: Multiple hyperpigmented papules on neck, upper chest, upper back, abdomen and upper arms coalescing centrally to form plaques and demonstrating reticulation peripherally.

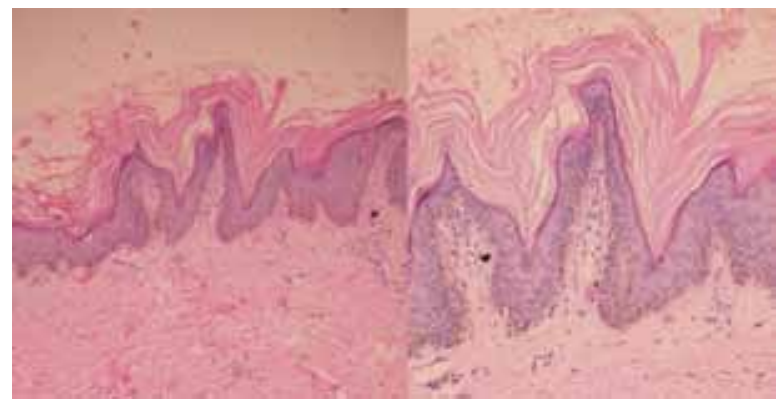

Figure 2: Skin biopsy showing hyperkeratotic epidermis with papillomatosis and mild perivascular lymphocytic infiltration in dermis (A, H and $E$ stain X100). High power view showing hyperorthokeratosis with mild acanthosis and slight hyperpigmentation of basal layer. (B, $\mathrm{H}$ and $\mathrm{E}$ stain, $\mathrm{X} 400$ )

On examination, there were multiple hyperpigmented papules on the upper chest, back, neck, upper arms and axillae, which had coalesced centrally to form plaques while demonstrating reticulation peripherally (Figure 1).

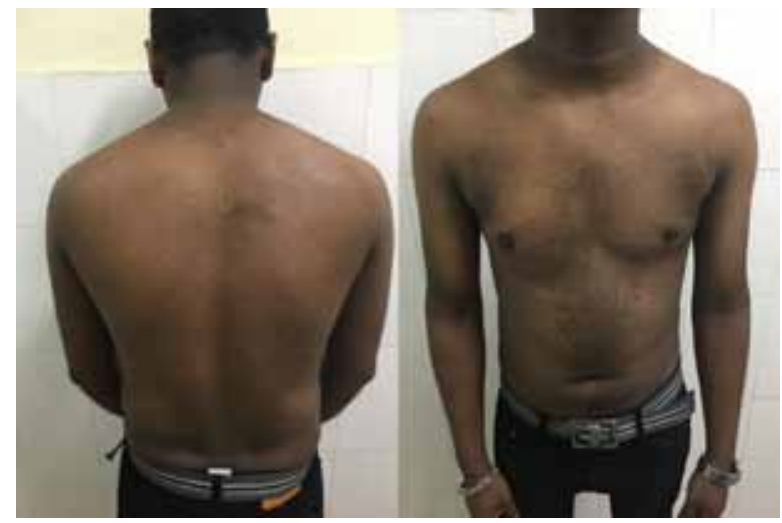

Figure 3: Resolution of the lesions on the neck, trunk and back with residual hyperpigmentation.

The lesions were swabbed with $70 \%$ alcohol, which did not lead to removal of the lesions. A potassium hydroxide test did not reveal presence of any fungal organisms. A biopsy taken from trunk revealed slight acanthosis with hyperorthokeratosis, papillomatosis, and slight hyperpigmentation of the basal layer. Underlying dermis showed mild degree of perivascular lymphocytic infiltration (Figure 2). Periodic acid Schiff staining did not reveal presence of any fungal organism. Congo red was negative for amyloid material.

On the basis of these findings, his condition was diagnosed as CRP and he was initiated on capsule minocycline $50 \mathrm{mg}$ twice daily along with nightly application of tretinoin $0.05 \%$ gel. There was complete resolution of the lesions after two months of treatment and he has remained disease free for the last six months (Figure 3).

\section{Discussion}

CRP usually occurs in young adults with asymptomatic brown to hyperpigmented papules on the upper trunk, axillae and neck. ${ }^{1}$ An infective aetiology was confirmed based on response of the condition to antimicrobials and the demonstration of Dietzia papillomatosis on the skin scrapings by Jones et al. ${ }^{2}$ It has also been associated with pregnancy, insulin resistance, obesity, pituitary and thyroid disorders and can also be familial. ${ }^{3}$

The diagnosis is based on the criteria developed by Davis et $\mathrm{al}^{4}$ in 2006, which is as follows: (i) presence of scaly brown macules and patches, some of which are reticulated and papillomatous; (ii) upper trunk and neck involvement; (iii) absence of fungus on skin scales; (iv) absence of response to antifungal treatment; and (v) excellent response to minocycline. The histological features suggestive of CRP are: 1) basket-weave orthohyperkeratosis, 2) papillomatosis, 3) focal acanthosis and 4) increased basal melanin pigmentation. ${ }^{1}$ 
The differentials that we had considered in our case were acanthosis nigricans, pityriasis versicolor, terra firma-forme dermatosis and Darier disease. Acanthosis nigricans was excluded on the basis of absence of increased body habitus and presence of acanthosis and dermal inflammatory infiltrates on histology. We ruled out pityriasis versicolor based on history of non-responsiveness to oral and topical antifungals and the absence of hyphae and spores on potassium hydroxide preparation of skin scrapings. We had swabbed his skin with $70 \%$ alcohol which did not lead to any improvement, thus ruling out terra firmaforme dermatosis. Darier disease was excluded based on absence of nail and palmoplantar changes and absence of characteristic histological findings.

The therapeutic options include oral antimicrobials, oral and topical antifungals, and topical retinoids and topical calcineurin inhibitors. ${ }^{1}$ We started the patient on minocycline $50 \mathrm{mg}$ BD for two months following which there was complete resolution of his lesions. He has remained disease free for the last 6 months. As one of the diagnostic criteria proposed by Davis et $\mathrm{al}^{4}$

\section{References}

1. Lim JH, Tey HL, Chong WS. Confluent and reticulated papillomatosis: diagnostic and treatment challenges. Clin Cosmet Investig Dermatol 2016;9:217. https://doi.org/10.2147/ CCID.S92051

2. Jones AL, Koerner RJ, Natarajan S, Perry JD, Goodfellow M. Dietzia papillomatosis $\mathrm{sp}$. nov., a novel actinomycete isolated from the skin of an immunocompetent patient with confluent and reticulated papillomatosis. Int J Syst Evol Microbiol 2008;58(1):68-72. https://doi.org/10.1099/ijs.0.65178-0

3. Henderson Berg $\mathrm{MH}$, Pehr K. Familial Confluent and Reticulated Papillomatosis in 2 Kindreds Including 3 Generations. J Cutan Med Surg 2018;22(3):330-2. doi: https://doi.org/10.1177/1203475417738968 was response to minocycline, our patient fulfilled all the criteria for CRP.

The other antimicrobials that can be used are doxycycline, tetracycline and azithromycin. The response to antibiotics is excellent with more than $50 \%$ response to minocycline or azithromycin. ${ }^{1}$ Cases that are unresponsive to minocycline or azithromycin can be initiated on systemic retinoids such as isotretinoin. ${ }^{5}$

Some authors believe that the condition is transitory, can resolve on its own and may not require active therapy. ${ }^{6}$ However, as our patient had the condition for 7 years and was slowly progressive, wait-and-watch may not be a feasible option in most cases.

\section{Conclusions}

CRP is a rare condition characterized by development of dark brown lesions on the trunk, neck and axillae of young adults. It should be kept in the differentials of non responsive pityriasis versicolor. The response to antibiotics is excellent and they should be initiated on all patients with the condition instead of adopting a wait-and-watch approach.

4. Davis $\mathrm{M}$, Weenig $\mathrm{R}$, Camilleri M. Confluent and reticulate papillomatosis (GougerotCarteaud syndrome): a minocycline-responsive dermatosis without evidence for yeast in pathogenesis. A study of 39 patients and a proposal of diagnostic criteria. $\mathrm{Br} J$ Dermatol 2006;154(2):287-93. https://doi.org/10.1111/ j.1365-2133.2005.06955.x

5. Hodge JA, Ray MC. Confluent and reticulated papillomatosis: response to isotretinoin. J Am Acad Dermatol 1991;24(4):654. https://doi. org/10.1016/S0190-9622(08)80164-8

6. Sakiyama T, Amagai M, Ohyama M. Chronology of confluent and reticulated papillomatosis: Spontaneous regression in a case after longterm follow-up may imply transient nature of the condition. J Dermatol 2015;42(3):335-6. https:// doi.org/10.1111/1346-8138.12776 